T. Gorschek, P. Garre, S. Larsson and C. Wohlin, "A Model for Technology Transfer in Practice", IEEE Software, Issue November/December, pp. 88-95, 2006. 


\section{A Model for Technology Transfer in Practice}

Tony Gorschek

tony.gorschek@bth.se

Blekinge Institute of Tecbnology

School of Engineering

PO Box 520

S-372 25 Ronneby, Sweden

Fax: +4645727125

Phone: +46457385817

\section{Per Garre ${ }^{\mathrm{i}}$}

per.garre@danahermotion.se

Danaher Motion Särö AB

42980 Särö, Sweden

Phone: +4631938125

Fax: +46 31938100

Stig BM Larsson ${ }^{\mathrm{i}}$

stig.bm.larsson@se.abb.com ABB Corporate Research

S-721 78 Västerås, Sweden

Phone: +4621345135

Fax: +46 21323212

Claes Wohlin ${ }^{\mathrm{i}}$

claes.woblin@bth.se

Blekinge Institute of Technology

School of Engineering

PO Box 520

S-372 25 Ronneby, Sweden

Fax: +4645727125

Phone: +46457385820

\footnotetext{
${ }^{\mathrm{i}}$ Authors stated in alphabetical order.
} 
The successful transfer of knowledge and technology from research to practice implies symbiosis. Close cooperation and collaboration between researchers and practitioners benefit both parties. Researchers get the opportunity to observe first-hand the challenges facing industry, making it possible for research not only to be based on interesting issues, but industry relevant ones. This entails on-site observations and process assessments, as well as collaboration with industry champions when designing and validating new methods, tools and techniques. Industry practitioners benefit as new technology (e.g. methods, tools and techniques) are developed based on tangible and real issues identified on-site, and step-wise collaborative validation helps minimize technology transfer risks and maximize potential benefit. This partnership between researchers and practitioners builds trust, and ultimately makes technology and knowledge transfer ${ }^{\text {ii }}$ possible.

We present a technology transfer process and experiences in using it. The process was devised and used during research conducted as a partnership between Blekinge Institute of Technology and two companies, Danaher Motion Särö AB (DHR) and ABB. The partnership was initiated with the intent of conducting industry relevant research in the area of requirements engineering. Technology transfer in this context is a prerequisite. From a research perspective technology transfer is needed to enable validation of research results in a real setting, while from an industry perspective technology transfer is a way to improve development and business processes.

From our experiences technology transfer (and industry relevant research) is not about producing

\section{Industry partners}

DanaherMotion Särö AB develops and sells software and hardware equipment for navigation, control, fleet management and service for Automated Guided Vehicle (AGV) systems. More than 50 AGV system suppliers worldwide are using DHR technologies and expertise together with their own products. The headquarters and R\&D centre is located in Särö, south of Gothenburg, Sweden with 85 employees.

$\mathrm{ABB}$ is a leader in power and automation technologies that enable utility and industry customers to improve performance while lowering environmental impact. The ABB Group of companies operates in around 100 countries and employs about 102,000 people. The transfer of new methods for requirement engineering was performed with one of the $\mathrm{ABB}$ development centers in Sweden. The product development part of this organization has 200 employees, including development and product management.

Both companies are participating in a joint long-term (6 year) research project with Blekinge Institute of Technology in the area of process improvement and requirements engineering. The collaboration in requirements engineering started in late 2002 with DHR, ABB joined late 2003.

\footnotetext{
ii We call it "technology transfer" or "transfer" for short.
} research results and handing them over in the form of publications and technical reports. The process we used involved several steps, one building on the other, carried out during a long-term joint commitment. These steps were devised in close collaboration with industry, and although inspired by previous transfer models, see e.g. Pfleeger ${ }^{1}$, it was created in an evolutionary manner, adding steps as needed. This evolution also dictated what each step of the process contained, e.g. how validation is performed depends on the needs of the company (what they trust), as well as the needs of the researchers to validate new technology for academic purposes. Taking industry preference into account when performing validation (as a part of technology transfer) is important for success ${ }^{2}$.

The contribution of this article is to present a technology transfer model that was devised ondemand in collaboration with industry and equally important, to report experiences and lessons-learned from two cases. 
Figure 1 gives an overview of our process divided into seven fairly straightforward steps, all which are relevant and interdependent for overall transfer success.

\section{Step 1 - Basing research agenda on industry needs}

We started by assessing current practices and observing domain and business settings, getting an overview of the demands posed on industry ${ }^{3}$. Observation of the real world before we formulated our research questions was considered natural, and it is also crucial for the improvement endeavor ${ }^{4}$. Without a strong connection to needs perceived on-site by the practitioners themselves commitment can be difficult to obtain.

During the assessments and observations (see Figure 1, step 1) several potential areas for improvement within the fields of product management and requirements engineering were identified. These were subsequently analyzed and prioritized according to perceived importance, and dependency $^{5}$. Dependency in this case meant that future improvements (needs) should be transferred in a certain order, avoiding "hitching the cart in front of the horse".

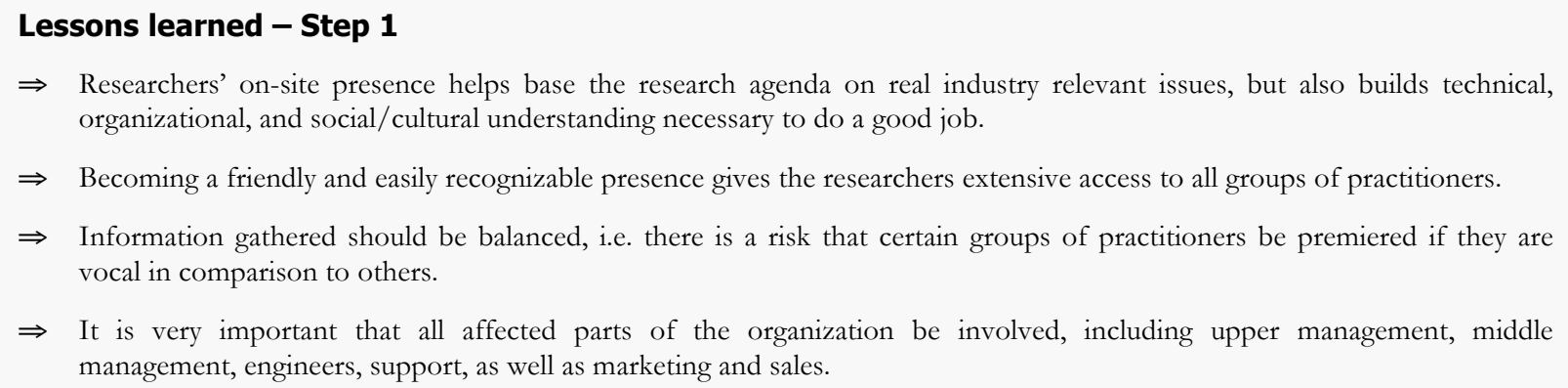

$\Rightarrow$ Researchers' on-site presence helps base the research agenda on real industry relevant issues, but also builds technical, organizational, and social/cultural understanding necessary to do a good job.

$\Rightarrow$ Becoming a friendly and easily recognizable presence gives the researchers extensive access to all groups of practitioners.

$\Rightarrow$ Information gathered should be balanced, i.e. there is a risk that certain groups of practitioners be premiered if they are vocal in comparison to others.

$\Rightarrow$ It is very important that all affected parts of the organization be involved, including upper management, middle management, engineers, support, as well as marketing and sales.

\section{Step 2 - Problem formulation}

Based on the prioritized needs a research agenda was formulated in close cooperation with industry contact persons, i.e. middle management practitioners. At this time these "contact persons" increased their committed and moved from participants to active "champions", contributing substantially with input and facts as well as making easy and fast access to the company organizations possible. The researchers also had a regular presence on-site, at first learning and observing, then as time went by more actively exchanging ideas and concepts with multiple practitioners, not only the champions. Doing your home-work and learning the domain establishes a common understanding and vocabulary. Close proximity between researchers and practitioners is considered a crucial factor for success ${ }^{4}$. 


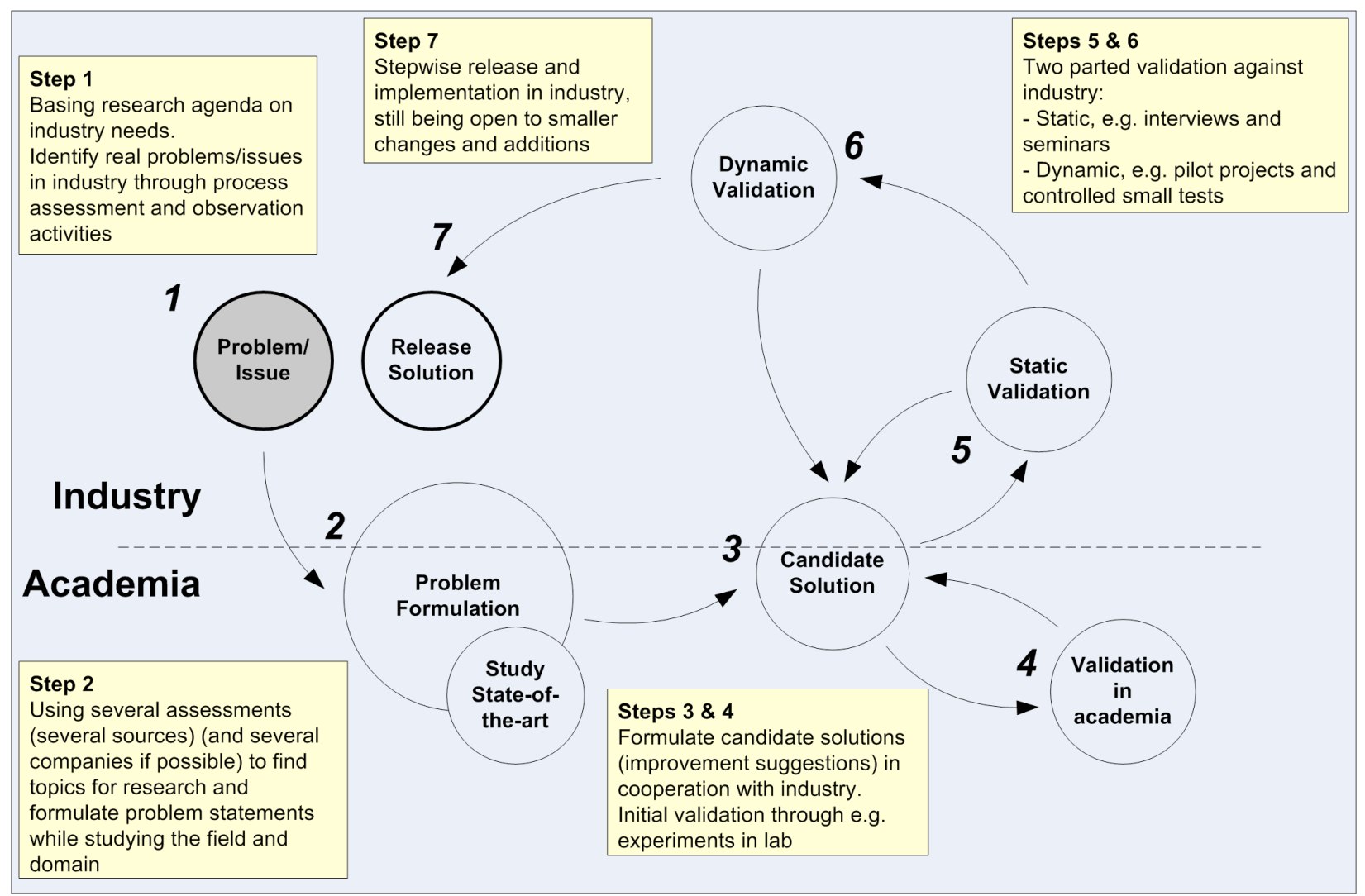

Figure 1. Research approach and technology transfer overview.

The main needs identified as high priority revolved around moving from bespoke "customerdeveloper" development to market-driven product-centered development. One of the main challenges in a market-driven environment is handling large volumes of requirements from multiple sources, both internal (e.g., developers, marketing, sales, support personnel and bug reports) and external (e.g., users, customers and competitors, often gathered via surveys, interviews, focus groups and competitor analysis) ${ }^{6}$. In this situation the volume of requirements to be handled makes initial screening important to decrease the risk of overloading in the evaluation and realization process ${ }^{7}$. In addition to this, the requirements themselves come in all different shapes and sizes, and on different levels of abstraction. Some of them very general and formulated like goals rather than requirements, while others very detailed and technical. There is a need to be able to handle all of them, make comparisons to product strategies and roadmaps, and to compare them in a prioritization situation. In our case both companies had similar needs, and thus a vested interest in addressing largely the same issues. 


\section{Lessons learned - Step 2}

$\Rightarrow$ Doing your "homework" implies learning company and domain specific vocabulary, as well as understanding practitioners' situation. This is needed to build trust.

$\Rightarrow \quad$ The role of the industry champion(s) is formalized and long-term commitment is obtained as practitioners take part in the problem formulation - which is the basis for the research agenda, i.e. what is to be done and improved.

\section{Step 3 - Formulate a candidate solution}

Subsequent to establishing a research agenda the collaboration with industry continued with the design of candidate solutions (improvements). Being specific, a requirement engineering model was designed (called RAM - The Requirements Abstraction Model) ${ }^{8}$. The model is intended to incorporate possible solutions for many of the needs identified during the assessments at DHR and $\mathrm{ABB}$, and primarily offers product planning and product management support. RAM is a multilevel requirements abstraction model, with a supporting process that aids practitioners in handling requirements in a structured and repeatable way, during requirements elicitation, analysis, refinement and management. The nature of the model is to use the fact that requirements come on different levels of abstraction instead of trying to flatten all or mixing different types in a document. Using RAM makes abstraction (comparing to strategies), and breakdown (until testable) of all requirements a part of the analysis and refinement work. As all requirements are abstracted and broken down it is also possible to compare them, making prioritization a realistic undertaking. (The RAM model itself is not detailed any further, for more information about RAM please see Gorschek and Wohlin ${ }^{8}$.)

The candidate solution was created in collaboration with practitioners. The main responsibility of the researchers was to keep track of state-of-the-art in research and apply this in combination with new ideas and angles. Another aspect of having practitioners as collaborators is the fact that they keep it real and compatible with the surrounding environment on-site. One problem often encountered as research is to be transferred is that solutions don't fit with the already present business/development methods ${ }^{9,10}$, increasing cost and effectively raising the bar for technology transfer.

\section{Lessons learned - Step 3}

$\Rightarrow$ Practitioners, in addition to being a resource, can provide a reality check, making sure that candidate solutions are realistic and to largest possible extent fit current practices and the situation at the company.

$\Rightarrow$ As candidate solutions are developed in collaboration with practitioners, commitment and trust are issues premiered. In addition the champions communicate and share ideas and information with colleagues, preparing for change in the mindset of the people in the organization.

$\Rightarrow$ Creating new solutions to issues identified is tempting. It is important that the researchers be the link to state-of-the-art in research, i.e. seeing to it that techniques, processes, and tools already developed and validated aren't ignored. In our case it meant building and refining some research results obtained by others, as well as invention of several new parts. 


\section{Evolution and transfer preparation through validation}

As the model (candidate solution) was formulated there was a need for evaluation. Several validation steps (and iterations of these steps) were used to accomplish this. The idea is to refine the candidate solution, test it for usability, scalability and if it actually addresses the needs in a satisfactory way. In addition, the validation steps are meant to gradually prepare for technology transfer. In this case both the solution itself needs to evolve (based on feedback from validation), but there is also a need to prepare the company. Preparation speaks to showing that there is a relative advantage to using the new solution in comparison to doing business as usual. This is crucial for getting commitment to transfer, something that researchers often miss?

\section{Step 4 - Lab validation}

First, the model was evaluated in a university lab environment prior to (and to some extent parallel with) static validation in industry (Figure 1, step 5). The evaluation was conducted in an experimental setting using master program students in software engineering as subjects. They were asked to use the model, performing requirements engineering activities as we envisioned them being performed in industry. The results from the evaluation made it possible to catch some issues without using industry resources. In addition we got early input as to the usability and scalability of the model. One motivation for early validation in academia is to ensure that pressure to transfer ${ }^{11}$ does not take the upper hand, i.e. trying to transfer all research results indiscriminately.

\footnotetext{
Lessons learned - Step 4

$\Rightarrow$ An initial practical test of the candidate solution in a lab environment can give valuable and fast feedback, i.e. finding (in hindsight) obvious flaws and fixing them prior to industry piloting.

$\Rightarrow \quad$ The results from a lab validation can be used when the candidate solution is presented widely to practitioners and management. Having initial results of using a candidate solution helps convince management of manageable risk and the potential benefit.

$\Rightarrow$ It is important to realize that lab validation is not the same as live industry usage. Realizing the limitations adds to the validity of the lab evaluation when presenting it to management in industry.
}

\section{Step 5 - Static validation}

Static validation (Figure 1, step 5) involved widespread presentation of the model in industry, and at the same time collecting feedback from practitioners. First, the model was presented to all industry personnel that were involved in the initial process assessment (Figure 1, step 1). Practitioners representing developers, designers, project managers, product managers, and sales \& marketing got to see the candidate solution through several seminars. In addition to getting feedback from practitioners (which further improved the model), the main goal was to give the practitioners feedback too. It was crucial to us that the practitioners that were to use the model got to voice their opinion early. Involvement and two-way communication between researchers and practitioners not only has the potential of improving results, but also laying the foundation for crucial issues such as 
shared commitment between researchers and practitioners, i.e. getting support for process change on all levels in the organization ${ }^{4}$. As an extension of the seminars several follow-up interviews were conducted.

The other main part of the static validation was presenting the research results and model to uppermanagement exclusively in a series of interactive seminars. Upper-management controlled resources needed for taking the next steps, basically implying that if they were not convinced of the benefit, the quality of the research could be irrelevant in terms of transferring it to practice. High quality research is not sufficient.

Management support is crucial ${ }^{4,10}$ and this fact cuts both ways. On the one hand, selling an idea (in this case the model) to management often refines arguments and can be a good control-point when it comes to devoting resources for technology transfer. On the other hand, at this early stage the research is unproven in real practice, thus it's hard to show objective facts (e.g. metrics) to motivate the transfer. These seminars also showed that the model had the support of operative personnel and middle management in the organization.

The bottom-line of the static validation was not just selling the model to industry representatives. The seminars and interviews gave invaluable feedback regarding usability and scalability of the model. For example about 15\% of the contents of the model (e.g. attributes for requirements, validation steps of requirements in the process etc.) were stripped away during this stage. What had worked in theory (and gotten past lab evaluations in step 4) needed to be slimmed down. Very early during the static validation we realized that a smaller model that produced good-enough requirements and decision support materials was preferable to a larger model that risked not being used at all.

\section{Lessons learned - Step 5}

$\Rightarrow \quad$ Widespread presentation of candidate solutions in the organization has several purposes: getting feedback and ideas for improvements, validating understanding and coverage, as well as giving feedback to the practitioners involved in the assessment phase in Step 1.

$\Rightarrow$ Anchoring future change (transfer) in all levels of an organization is crucial.

$\Rightarrow$ Upper-management seminars and interactive sessions are crucial to assess risk and potential relative benefit of the changes proposed through the transfer of the candidate solution. It is important to show that the candidate solution was developed in cooperation with and has the support of practitioners in the organization.

$\Rightarrow \quad$ It is very important that researchers are not afraid of tailoring (even some down-scaling) the candidate solution at this stage. Change should be seen as a part of the candidate solution validation and refinement, and is natural as the ideas presented mature and grow over time.

\section{Step 6 - Dynamic validation (piloting)}

Dynamic validation was performed through two pilot projects, both performed at DHR. The first one was limited in scope, using the model to elicit, analyze, refine, and manage about 75 requirements in total. The work in this first pilot was carried out by the researchers and an industry project manager (champion). The product being specified was an in-house product configuration tool and the customers were also situated in-house. 
The success of this first pilot gave motivation and commitment by the company to continue the dynamic validation with a larger pilot. The second pilot was carried out using the model in a real development project, without the direct involvement of the researchers or industry champions, although the model used in the first pilot was reused to some extent. The project had 18 developers and ran for about 4 months (3500 person-hours). The project manager was the primary requirements engineer; the developers used the requirements (produced with the model) in their work.

Both pilots gave valuable feedback, although not many new critical problems or potential difficulties were reported. The primary feedback we got was that tool support, as well as tool adaptation was important for model scalability. In addition there was a need to incorporate training efforts as a part of future model implementation. The main reason for there not being any big "surprises" during piloting was felt to be due to the static validation work and the continuous collaboration between the researchers and the industry champions. The relative success of the dynamic validations increased the credibility of the model, and built trust for us working with the model (researchers and champions), in the eyes of practitioners and management.

\section{Lessons learned - Step 6}

$\Rightarrow$ Piloting in industry gives the possibility to perform realistic evaluations of candidate solutions without giving up control (minimizing risk as the pilot is a limited test).

$\Rightarrow$ Pilots can be used to get input for further improvements, and indications of what is needed during the full scale transfer.

$\Rightarrow$ As pilots are limited in scope they may not catch all potential problems like scalability issues.

$\Rightarrow$ As pilots to some extent precede "proper" transfer (e.g. training is not formalized and the solution itself is not released) the usage of the candidate solution may differ somewhat from what was intended.

\section{Step 7 - Release solution}

Gauging the results from the validations (static and dynamic) a decision was made to give the goahead with the actual implementation of the model, i.e. official release. At DHR the model was to be implemented fully and incorporated in the official development and management process. At ABB the implementation was trialed and initially limited to one large release project, full implementation at $\mathrm{ABB}$ pending results from this "trial-release". Despite of this difference, the technology transfer process and the techniques used to accomplish it were largely the same for both companies. It consisted of a tailoring of the model to fit each company specifically. RAM had been refined through the validations and pilots, but it was rather general in nature, a sort of least common denominator suiting both companies. A central part of the rationale behind the model was that we knew that one-size-does-not-fit-all, an important lesson on research from working with two companies. Thus RAM can be tailored to fit different organizations taking into account e.g. differences in types of requirements, specific domains, and so on, catering to a specific organization's needs. 


\section{Model tailoring}

Tailoring was necessary for several reasons. The two companies had different definitions and vocabulary pertaining to products and development, and specific practices and processes already in place had to be taken into account to align the RAM model for transfer into a specific organization.

\section{One day workshop - tailoring and consensus}

The first stage in model tailoring consisted of a workshop conducted at each company over one entire day. Management, developers, and especially product and project managers (future primary users of the model) participated. Process owners were also present. The focus was on adapting the model not only to suit current practices, but e.g. roles and responsibilities set forward by the model were mapped to existing ones where possible. Concepts like good-enough were explored, establishing some examples of requirements through actual specification of requirements during the workshop.

The requirements that were jointly specified, refined and analyzed (using the model) were taken from within the company keeping it as "real" as possible. As we worked with the example requirements the researchers and the industry champion took turns, one leading the workshop and the other documenting results. The work with requirements proved to have a grounding-effect, keeping things tangible and discussions from becoming too abstract and theoretical.

Obtaining consensus among all groups represented and assuring that compromises made didn't impede usability, was the main objective of the workshop - thus also promoting future commitment.

\section{One week alignment - formalization and planning}

The information gathered during the workshop was crucial for the formalization and planning of the model implementation into the official development and business process. In short this meant converting workshop results into practice, starting with the development of needed documentation, user guides, example databases of requirements (reusing requirements from the workshop and creating additional ones), and tool support. Very early in the process of developing and validating the model itself we realized that a document heavy, large, and complex model demanding too much from the practitioner may have been transferred to the official development and business process, but it would probably stay on the shelf collecting dust, next to other heavy frameworks and certifications.

Documentation consisted of two things that we separated to the largest possible extent. Formal documentation implied creating all mandatory official process descriptions, such as role descriptions, process maps, detailed requirement state diagrams, mapping to current processes, and glossaries etc. These official documents were needed, primarily as reference material, publicly accessible on a needto-use basis. The user documentation was most important. It consisted of a very thin (fewer than 5 pages) quick reference guide.

Training was like in most process improvement efforts considered very important ${ }^{9,10}$, and we prioritized it. A positive spin-off effect of the collaborative research process utilized in general was 
that many aspects of the model were already to some part assimilated by practitioners in the companies as many of them had a part in creating or validating them. This meant that training was made easier, not least in terms of commitment.

The main parts of the training were performed utilizing a learning-by-doing strategy. The champion led training sessions where one or two practitioners used the model to engineer real new requirements. We nicknamed this "pair-requirements engineering" (as in pair-programming). The idea behind it was twofold; first as a good way of learning and getting experience, second, by performing actual work during training sessions the cost of training would decrease.

Example driven was one of the main points with the model and thus the training. The company specific (relevant) requirements developed during the workshop and the alignment week were used as good-practice examples (notice that we don't use "best-practice"). The examples were in themselves an important part of the "documentation", i.e. practitioners could use them in their training and later in their day-to-day work as an information source. For this reason we tried to categorize some different types of requirements (most common), and create a database of examples of each category.

Tool support was important. During the week we adapted the tool chosen by respective company. As the model is a way of working with requirements, it is largely tool-independent. This was evident as the companies chose to use different tools to support the model.

Technical support is a factor that can greatly influence the success of a technology transfer effort ${ }^{11}$. It is important that practitioners have access to continuous support from an expert, or at least an expert-user, enabling continuous support as needed. Practitioners frustrated and stuck need help fast, or there is a risk that they go back to performing requirements engineering as they did previously. Both DHR and ABB have champions filling the role of expert.

Owner and champion is a role to some extent shared by the researchers and the champions during the research and validation of the solution candidate. However, as the candidate evolves and is released ownership has to be (and was) transferred to the company, and relevant champions on-site. This is necessary as the researchers cannot be present and active in supporting practitioners to the extent necessary. Optimally, with time and usage the model will be owned and championed collectively by all practitioners using it. This is however not the same as saying that the researchers' presence or participation is at an end, rather the opposite, as follow-up studies have to be performed.

Metrics, i.e. the collection of data regarding the usage of the model is a crucial part of a technology transfer process enabling measurement and follow-up studies. During the alignment week a plan for how to measure (what metrics to collect) was formulated. It was important to enable measurement of the model usage for several reasons. First, after a time metrics collected could indicate benefit and/or hint at potential problems. 
In addition to quantitative measurement qualitative follow-up was also planned, e.g. interviews and group discussions. The combination of qualitative and quantitative follow-up is intended to provide feedback for future changes and modifications.

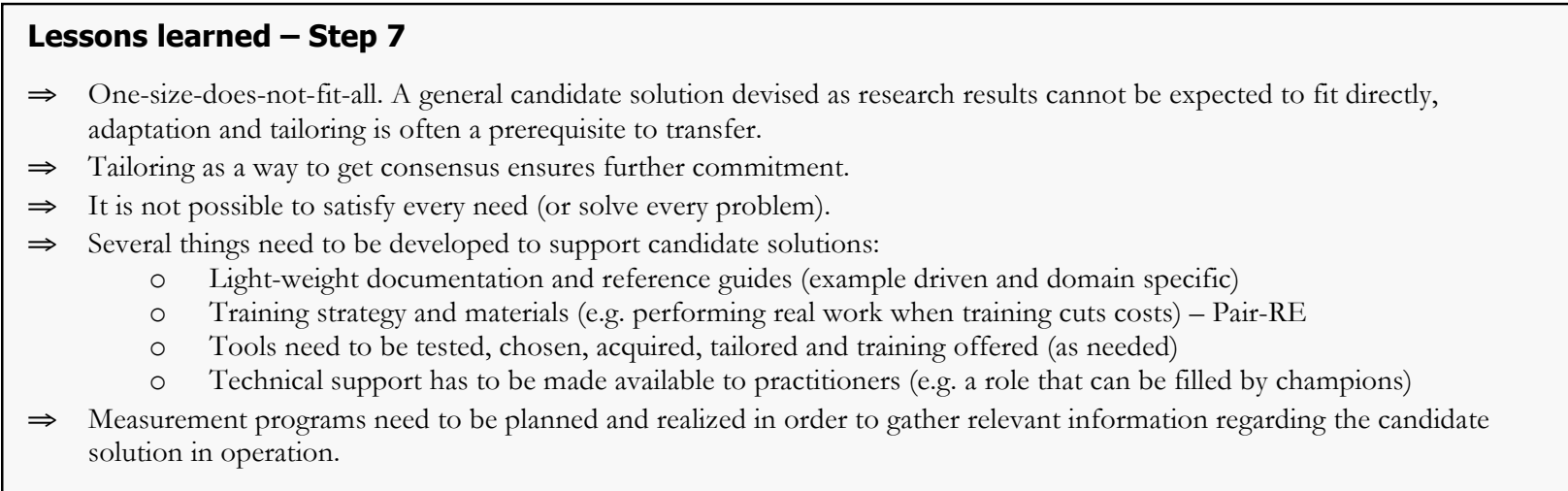

\section{Process observations}

The actual process used was formulated in close collaboration between the researchers and the industry partners. This was crucial to obtain commitment and trust from everybody involved. However, it is interesting to note that the actual process formulated for the two companies in Sweden resembles the levels presented as the Technology Readiness Levels ${ }^{12}$ as formulated by government bodies in the US. This implies that there is a growing consensus regarding which steps or levels are needed for successful transfer and adoption new technology.

\section{Conclusions in perfect hindsight}

Looking back several issues come to mind as "critical success factors". Commitment, having champions, collective ownership, building trust, and training are all examples. We will not try to cover all of them here, rather mention a few things in addition to the experiences shared this far - all in perfect hindsight of course.

\section{Long-term commitment - Short-term-long-term benefits}

Neither research nor technology transfer is a one-shot deal. It requires long-term commitment from both researchers and practitioners. Technology transfer happens over time as small bits break-off and is adopted by practitioners continuously. This can be frustrating, from a research perspective, as it is hard to measure the impact of such "improvements". The collaborative research process itself can be seen as implicit technology transfer under favorable conditions.

Long-term in this case applies to the lead-time required. This is partially due to that practitioners perform their daily work in addition to working with the researchers. This is not the same thing as lack of commitment, rather that practitioners are pressed for time as they operate in a competitive 
environment ${ }^{11}$. Performing lab validations prior to industry trials is a good idea as it can help catch issues without consuming resources.

\section{Risk minimization}

The ability to maximize potential benefit and minimize the potential risk is of paramount importance to companies. The validation in iterations improved the model and helped us convince management and practitioners (and ourselves) that the risks were acceptable in comparison to the potential benefit. This said, the researchers' job isn't just research, its making transfer happen. Active participation in some activities that by themselves don't produce research results (papers) can be considered as overhead from a research perspective, but a necessity from an industry perspective.

We were fortunate in our endeavors as both researchers and practitioners have a pragmatic take on things. Research results were considered important, but from our point of view the value of these results were directly linked to usability in industry.

\section{Technology transfer}

The model used for technology transfer was not prepackaged. The steps and the contents of each step were added on-demand to be appropriate for the situation. In some cases this meant additional work to provide sufficient evidence for e.g. management showing the relative value of a new way of working. In terms of future research this suggests flexibility - on-demand modification and addition of steps and iterations to satisfy both industry demands of risk minimization and relative value evidence, as well as the needs of the researchers. Our technology transfer model should be seen as an instantiation of a technology transfer process, which can be used for inspiration rather than prescription.

\section{References}

1. S. L. Pfleeger, "Understanding and improving technology transfer in software engineering", Journal of Systems and Software, vol. 47, no. 2-3, 1999, pp. 111-124.

2. $\quad$ S. L. Pfleeger and W. Menezes, "Marketing technology to software practitioners", IEEE Software, vol. 17, no. 1, 2000, pp. 27-33.

3. T. Gorschek and C. Wohlin, "Identification of Improvement Issues Using a Lightweight Triangulation Approach", Proceedings of European Software Process Improvement Conference (EuroSPI'2003), Verlag der Technischen Universität, 2003, pp. VI.1-VI.14.

4. V. R. Basili, F. E. McGarry, R. Pajerski and M. V. Zelkowitz, "Lessons learned from 25 years of process improvement: the rise and fall of the NASA software engineering laboratory", Proceedings of the 24th International Conference on Software Engineering (ICSE02), ACM, 2002, pp. 69-79.

5. T. Gorschek and C. Wohlin, "Packaging Software Process Improvement Issues - A Method and a Case Study", Software: Practice \& Experience, vol. 34, no. 14, 2004, pp. 1311-1344. 
6. D. R. Lehmann and R. S. Winer. Product management. McGraw-Hill, 2002.

7. M. Weber and J. Weisbrod, "Requirements Engineering in Automotive Development:

Experiences and Challenges", IEEE Software, vol. 20, no. 1, 2003, pp. 16-24.

8. T. Gorschek, "Software Process Assessment \& Improvement in Industrial Requirements

Engineering", School of Engineering - Department of Systems and Software Engineering, Blekinge Institute of Technology, 2004, pp. 154.

(http://www.ipd.bth.se/tgo/_licentiate/papers/Licentiate)

9. P. Morris, M. Masera and $\bar{M}$. Wilikens, "Requirements engineering and industrial uptake", Proceedings of the Third International Conference on Requirements Engineering, IEEE, 1998, pp. 130-137.

10. S. Miller, "How can Requirements Engineering Research Become Requirements Engineering Practice?" In Proceedings of the Third IEEE International Symposium on Requirements Engineering, IEEE, 1997, pp. 260.

11. H. Kaindl, S. Brinkkemper, J. A. Bubenko Jr, et al., "Requirements Engineering and Technology Transfer: Obstacles, Incentives and Improvement Agenda", Requirements Engineering, vol. 7, no. 3, 2002, pp. 113 - 123.

12. J. C. Mankins, "Technology Readiness Levels - A White Paper", Office of Space Access and Technology, NASA, 1995, pp. (http://advtech.jsc.nasa.gov/downloads/TRLs.pdf) 